\title{
Clinical spectrum of ear, nose and throat foreign bodies in North Western Nigeria.
}

\author{
Taiwo Olugbemiga Adedeji ${ }^{1}$, Olusola Ayodele Sogebi ${ }^{2}$, Sahabi Bande ${ }^{3}$
}

\author{
1. LAUTECH Teaching Hospital, Osogbo, Ear, Nose and Throat. \\ 2. Olabisi Onabanjo University Teaching Hospital, Ear, Nose and Throat. \\ 3. Federal Medical Center, Birnin Kebbi, Ear, Nose and Throat.
}

\begin{abstract}
Background: Ear, nose and throat foreign bodies (FBs) are common occurrences particularly among children. This study reviewed the clinical spectrum of ENT FBs, their treatment and outcomes as seen in a tertiary health center in North Western Nigeria.

Method: The study was a retrospective chart review of patients that were managed for FB impaction in a tertiary health institution in North Western Nigeria over a four year period.

Result: There were 239 patients; M: F: 1.2:1. Majority of FB impaction (46.4\%) occurred in children. Majority (68.7\%) were otic and FBs. $18.0 \%$ of the patients had had failed attempted removal by non ENT specialists. About $25 \%$ of these patients developed complications. Majority $(62.0 \%)$ of these complications occurred in the hand of non-ENT medical personnel.

Conclusion: Ear, nose and throat foreign bodies are common in North-Western Nigeria with the highest incidence in children. Removal attempts by untrained health professionals and lack of experience in FB management predisposes to complications. Parental education on close monitoring of their children to avoid such incidences and the need to immediately seek an Otorhinolaryngologist to prevent complications are emphasized.
\end{abstract}

Keywords: Craniofacial orifices, foreign bodies, clinical spectrum, management, Nigeria

DOI: http://dx.doi.org/10.4314/ahs.v16i1.38

Cite as: Adedeji TO, Sogebi $O A$, Bande S. Clinical spectrum of ear, nose and throat foreign bodies in North Western Nigeria. Afri Health Sci. 2016;16(1): 292- 297. http://dx.doi.org/10.4314/abs.v16i1.38

\section{Introduction}

Ear, nose and throat (ENT) foreign bodies (FBs) are common occurrences and form a major part of emergencies that the Otorhinolaryngologist needs to attend ${ }^{1,2,3}$. A foreign body is an object which lodges into a craniofacial orifice which includes the ear, nose, or throat. It is particularly common in the paediatric population especially below 5 years of age $e^{1,2}$ and in whom prevalence was reported to vary between 57 and $80 \%$ ${ }^{6}$. This high prevalence can be attributable to the inquisitive nature of children and their tendency to explore the environment ${ }^{1,4}$. Foreign body aspiration in adults is often accidental ${ }^{1,2}$, and it is also encountered in some mentally deranged adults. Cases of unusual foreign bodies deliberately swallowed for ritual purposes have also been reported ${ }^{1}$.

Foreign bodies can be organic or inorganic. Organic foreign bodies have a tendency to elicit inflammatory

\section{Corresponding author: \\ Taiwo Olugbemiga Adedeji, LAUTECH Teaching Hospital, Osogbo, Ear, Nose and Throat. Email: adedejitaiwo2003@yahoo.com}

reactions ${ }^{5}$. In the ear, they predispose to otitis externa, suppurative otitis media and hearing loss $^{6}$. FB impaction in the nose predisposes to infective rhinosinusitis, foreign body granuloma and septal perforation. In the throat, there is tendency to peritonsillar and paratonsillar abscess, dysphagia and sometimes acute upper airway obstruction. These consequences are more grievous, if the affected child did not volunteer the history of FB ingestion or aspiration, if the FB elicits an inflammatory reaction by nature, if it is impacted along the airway or if there is no required expertise for its removal. FB impaction in the larynx is particularly dramatic and often presents as an emergency.

Various methods of FB removal have been described. In the ear the most commonly used method for removal is by syringing, while other instruments like forceps, fine hook, hair clip and suctioning can also be used. Live insects are first killed by drowning in methylated spirit followed by syringing ${ }^{6,7,8}$. In the nose; removal is accomplished by the use of wax hook, forceps or eustachian tube catheter ${ }^{3,7}$. In the throat, FB removal is accomplished by grasping with forceps while in the larynx and in the oesophagus removal is usually done under general anaesthesia ${ }^{7-11}$. 
It is necessary for physicians to be conversant with the common FBs ingested or aspirated in their communities of practice, and be prepared to render adequate and effective management, prevent complications and reduce morbidities ${ }^{6,7}$. This study aimed to answer the research question on what the clinical spectrum; types and distribution of ENT FBs were, in a tertiary health center in North Western Nigeria. It also evaluated their treatment and outcomes in this center.

\section{Material and methods}

The study was a retrospective chart review of patients that were managed for ENT foreign body impaction at Otorhinolaryngology Department Federal Medical Center, Birnin Kebbiover a four year period between December 2008 and November 2012. The case notes of the patients were retrieved from the medical records department of the hospital. Data retrieved from the case records by the investigators included patients' age, sex, type of foreign body and the site/side of impaction, methods of removal, cadre of medical personnel that removed the foreign body and outcome.

Excluded were patients whose case records could not be located and those that had incomplete information.
Ethical approval was waived for this study since it involved retrospective retrieval of information from case records of patients on the provision that confidentiality will be maintained.

The data was entered into a spread sheet to generate data that was statistically analyzed using statistical package for social sciences version 14 (SPSS 14). The data was presented in simple descriptive terms as proportions in tables and graphic chart.

\section{Results}

A total of 4,162 patients were seen during the study period, $239(5.7 \%)$ of whom had foreign bodies in the ear, nose and throat and presented for removal. FB removal represented $18.2 \%$ of the total ENT procedures done during the period under review. There were 132 males $(55.2 \%)$ and 107 females $(44.8 \%)$ with sex ratio 1.2:1 (M: F). The age of the patients ranged from 1 year to70 years (Mean $\pm \mathrm{SD}=12.8 \pm 14.1)$. The major proportion of the F.Bs impactions $(46.4 \%)$ were found in children less than 5 years of age, followed by children 6 - 10 years $(15.9 \%)$ while the least F.B $(0.84 \%)$ were found in the age group 61- 70 years. The age distribution of the patients according to sex is shown in Figure 1.

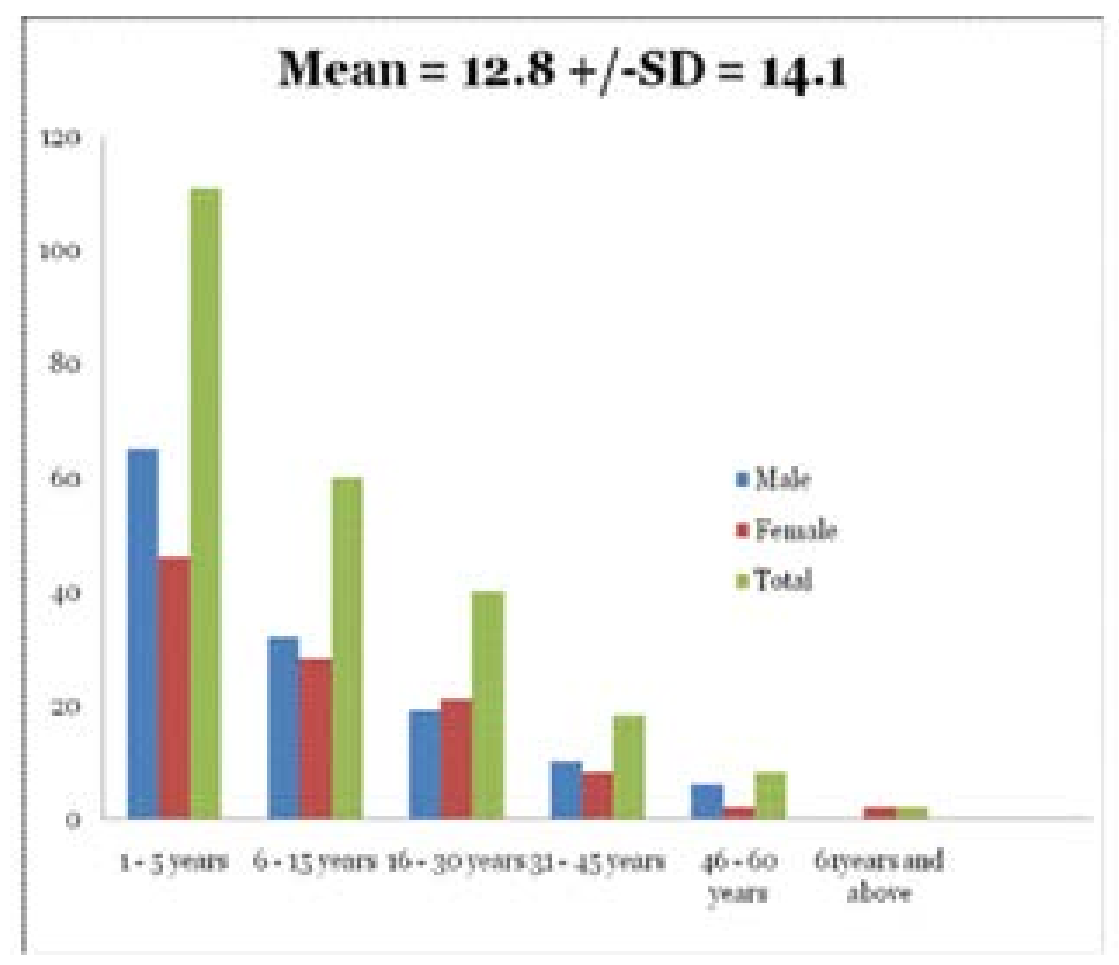

Figure 1: Age and sex distribution of patients.

There were $164(68.7 \%)$ cases of ear (otic) FBs; nose (nasal) FBs were $50(20.9 \%)$ while throat (pharyngo-la- ryngeal) FBs were 25 (10.5\%). Table 1 shows the distribution of the foreign bodies by site/side. 
Table 1. Location of foreign bodies by Site/Side

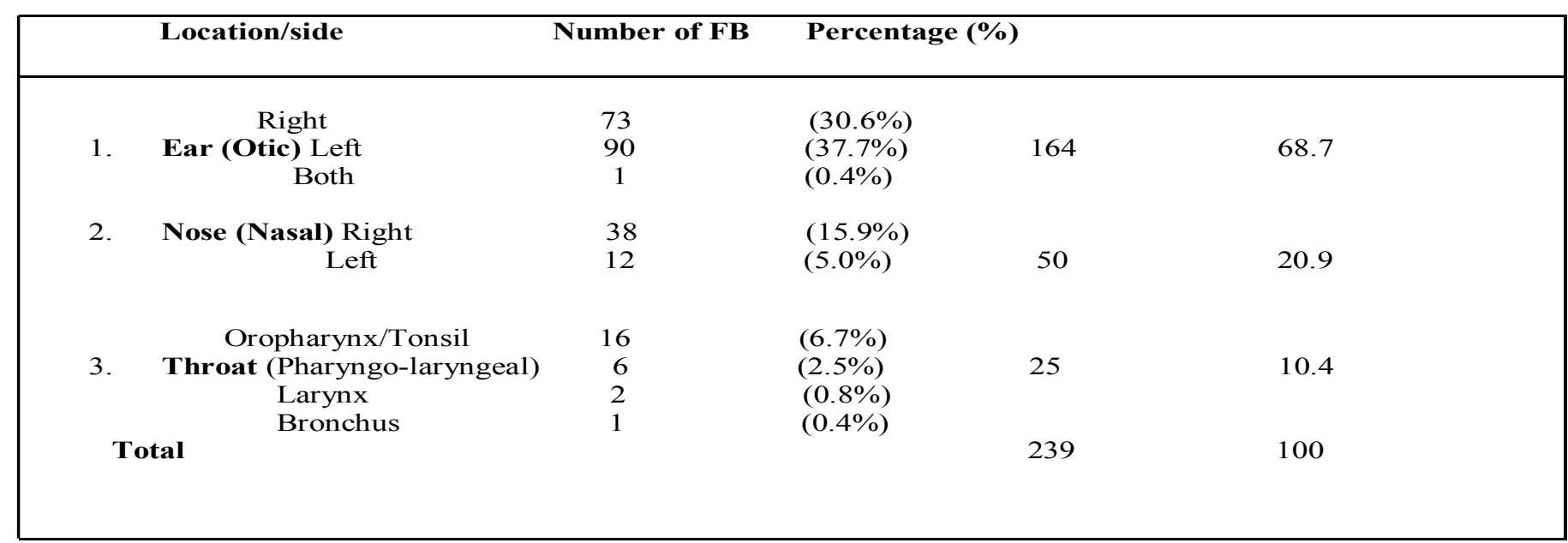

The most common foreign body removed from the ear, nose and throat were seeds (corns/beans/rice husk) totaling $47(19.67 \%), 42(17.6 \%)$ of these were found in children. Table 2 shows the distribution of the foreign bodies according to anatomical sites of impaction. Seven out of the 45 cotton buds were found in children 10 years and below. Majority of the beads $(90.0 \%)$ were found in the children and beads constituted the predominant $(30.0 \%)$ nasal foreign body.

Table 2. Types of FBs removed from the ear, nose and throat according to anatomical sites

\begin{tabular}{|l|cllc|}
\hline Foreign bodies & \multicolumn{4}{|c|}{ Site } \\
\cline { 2 - 5 } & Ear 164(\%) & Nose 50 (\%) & Throat 25(\%) & Total 239(\%) \\
\hline & & & & \\
Insect & $17(10.4)$ & $0(0)$ & $0(0)$ & $17(7.1)$ \\
Corn/Seed/Rice husk & $35(21.3)$ & $11(22)$ & $1(4)$ & $47(19.7)$ \\
Bead & $25((15.2)$ & $15(30)$ & $0(0)$ & $40(16.7)$ \\
Cotton bud & $45(27.4)$ & $0(0)$ & $0(0)$ & $45(18.8)$ \\
Foam & $0(0)$ & $6(12)$ & $0(0)$ & $6(2.5)$ \\
Chalk & $3(1.8)$ & $2(4)$ & $0(0)$ & $5(2.1)$ \\
Paper /Tissue & $11(6.7)$ & $3(6)$ & $0(0)$ & $14(5.9)$ \\
Fish bone & $0(0)$ & $0(0)$ & $14(56)$ & $14(5.9)$ \\
Stone & $8(4.9)$ & $5(10)$ & $0(0)$ & $13(5.4)$ \\
Glass & $1(0.6)$ & $0(0)$ & $0(0)$ & $1(0.4)$ \\
Match stick & $3(1.8)$ & $0(0)$ & $0(0)$ & $3(1.3)$ \\
Soap & $1(0.6)$ & $0(0)$ & $0(0)$ & $1(0.4)$ \\
Button & $1(0.6)$ & $1(2)$ & $0(0)$ & $2(0.8)$ \\
Rubber /plastic & $4(2.4)$ & $4(8)$ & $7(28)$ & $15(6.3)$ \\
Crayon & $2(1.2)$ & $0(0)$ & $0(0)$ & $2(0.8)$ \\
Key/metal & $0(0)$ & $0(0)$ & $3(12)$ & $3(1.3)$ \\
Others & $8(4.9)$ & $3(6)$ & $0(0)$ & $11(4.6)$ \\
Total & $164(100)$ & $50(100)$ & $25(100)$ & $239(100)$ \\
& & & & \\
\hline
\end{tabular}

All the FBs from the ear and nose were successfully removed in the clinic without the need for general anaesthesia. Live insects were killed first by drowning in olive oil before eventual ear syringing.

Out of $25 \mathrm{FBs}$ in the throat, 9 patients (with foreign body in the larynx, oesophagus and bronchus) had their foreign bodies removed under general anaesthesia via rigid endoscopy; others (with foreign body in the tonsil/oropharynx)were removed in the clinic with grasping forceps after the tongue had been depressed with a tongue depressor under proper illumination with head lamp or with head mirrors that directed light to the oropharynx. 
Eighty five percent of the FBs in the ear and nose were removed by ENT trained nurses, 7\% were removed by general duty doctors and $8 \%$ were removed by ENT surgeons. All the FBs that were removed under general anaesthesia were removed by ENT surgeons.

Eighteen percent of the patients had failed attempt at removal by medical workers that were not ENT trained before referral to ENT department for removal. About $23 \%$ of these patients had complications including epistaxis, unilateral offensive nasal discharge, abrasion/ laceration of the external auditory canal, otitis externa. Majority $(62 \%)$ of these complications occurred in patients that had attempted removal before referral to ENT department.

Figure 2: Complications among the patients with ENT FBs.

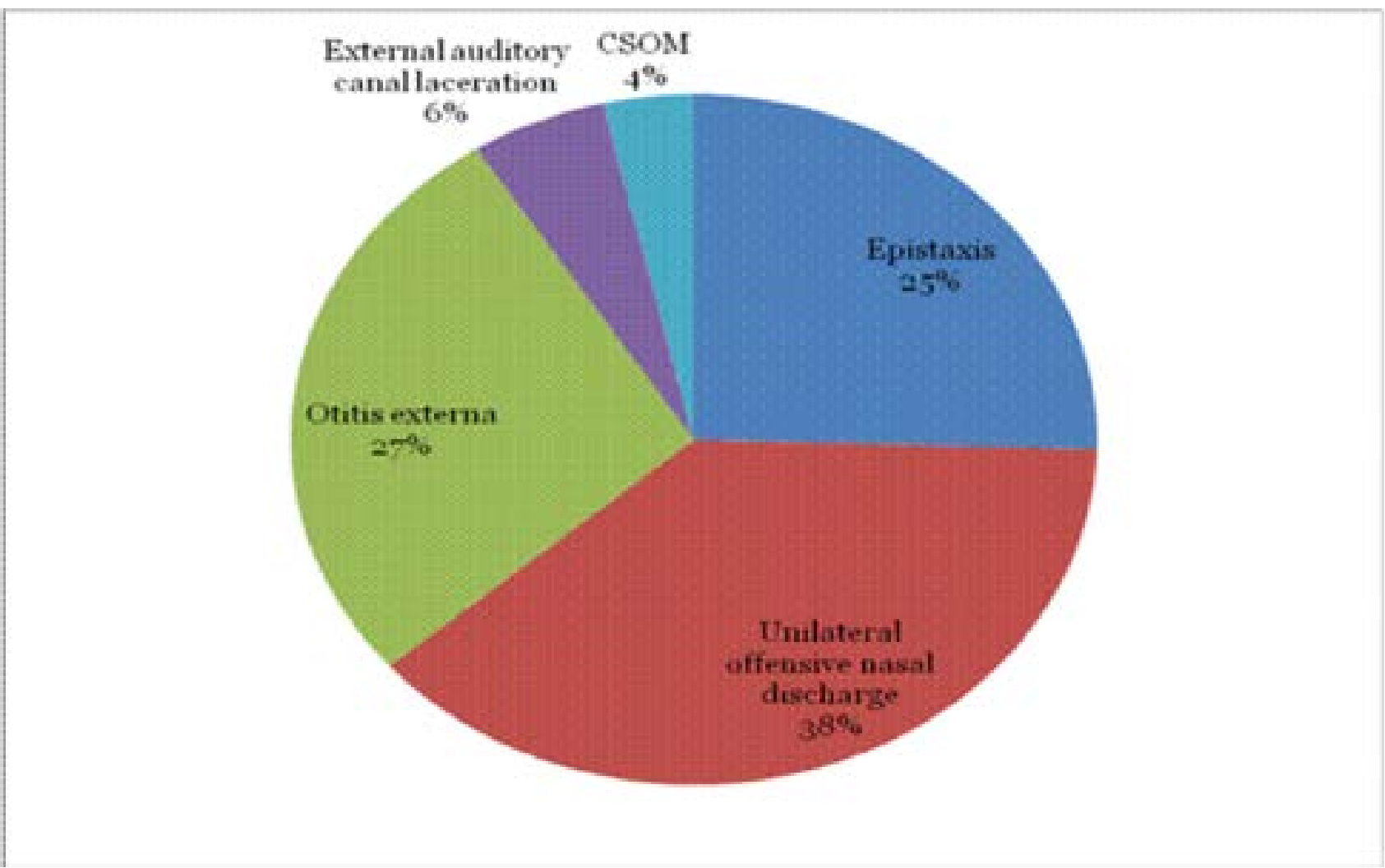

NB: CSOM Chronic suppurative otitis modia

Most of the complications cocurred from the peripheral referral centers where there were no ENT trained specialists-

\section{Discussion}

The incidence of foreign body impactions in the ENT region of $5.7 \%$ indicates that it is common in our locality. This is similar to the $6.3 \%$ reported by Ette et al in $\mathrm{Uyo}^{7}$ and resonates with the previous reports that ENT FBs are common and represent one of the challenging emergencies Otolaryngologists confront in their day to day practice ${ }^{1,6,8,9}$. Almost two-third $(62.3 \%)$ of the affected patients were children with ages 10 years and below and the prevalence was disproportionately high $(46.4 \%)$ in children under the age of 5 years. Other studies reported similar findings ${ }^{2,4,7,10}$. Parental carelessness, increases in bodily activities, explorative and inquisitive nature of the children are some of the reasons that had been adduced for this finding. ${ }^{11,12}$. This study also showed marginal male preponderance $(\mathrm{M}: \mathrm{F}=$ 1.2:1). This agrees with previous studies ${ }^{8,11}$ possibly because male children are likely to be more explorative by nature compared with their female counterparts. However Ette et al in Uyo reported female preponderance ${ }^{7}$. Parents and caregivers need to monitor their children closely and also remove potential FBs from the environment. They should also be encouraged to present their children early to hospital whenever they observe any unusual symptoms in them.

Ear foreign bodies were the most common (68.7\%), followed by nasal foreign bodies $(20.9 \%)$ in this study. This pattern has also been reported by others ${ }^{7,11,12 .}$ The external auditory canal is a cul de sac, the most narrow among the craniofacial orifices, and serpentine in con- 
figuration; these predispose to difficulty in extraction of impacted FBs. Unlike in the ear, foreign bodies are more easily extracted from the nose and throat sometimes by protective physiological mechanisms and reflexes like sneezing, nausea and coughing ${ }^{12}$.

More FBs were found in the ear in this study. However there is no consistency in reports about the more common side of FB impaction in the ears.

Cotton buds and seeds were the most common otic FBs; these have tendencies to elicit inflammatory reactions ${ }^{5}$. Cotton bud impaction is particularly common among the adults due to the almost universal practice of "cleansing" the ears. Unfortunately this is counterproductive, because it disrupts the natural ear cleaning mechanism by epithelia migration which is located along the walls of the external auditory canal (EAC). There is need for health education which should include persuasion of people to desist from cleaning the ear with cotton bud as the cotton tip can easily detach and impact into the ear canal.

Live insects present in a dramatic way with otalgia, restlessness and great discomfort which can confuse an inexperienced physician. However management involves killing and immobilizing the insect through drowning in oil, and subsequent removal of the dead insect by other means like manual extraction, or ear syringing. Impaction of alkaline battery button in the ear also carries the particular risk of leakage of the electrolytes which can cause liquefactive necrosis of the epithelium and surrounding tissues. Therefore it should be managed urgently with extraction using a magnet or any other means of manual removal. Battery buttons should not be syringed. Fortunately, this FB impaction is not common in our locality.

Foreign bodies seen in the nose in this study were mostly found in children (98\%). These findings are in agreement with the literature ${ }^{7,12}$. Beads are the commonest foreign bodies in the nose. Beads were found in many homes as religious symbols in rosaries and for cultural use $^{3}$ being worn as ornaments in the hairs or on the neck. ${ }^{6}$ The most common site of insertion was the right nasal cavity, similar to other findings ${ }^{3,7}$ and consistent with the right hand dominance in majority of people. Bead is supposedly inert and may be responsible for low incidence of mucopurulent nasal discharge in the affected children. However prolonged stay of the FB in the nasal cavity still predispose to infections. The most common manner of presentation is unilateral offensive nasal discharge. Common complications like rhinosinusitis are found in such patients but less common ones like foreign body granuloma and septal abscess and perforation can also occur. The key to adequate management remains a good history, high index of suspicion and appropriate technique for its removal.

Most of the FBs in the throat (64\%) got impacted in the oropharynx especially in or around the tonsils. Anatomic location of the tonsils makes it the most favoured site for impacted pharyngeal foreign bodies ${ }^{12}$. Majority of these oropharyngeal FBs were fish bones. Ette et al also reported similar findings ${ }^{7}$. Ahmad ${ }^{13}$ in Maiduguri over a decade earlier reported coins as the commonest FBs in the throat followed closely by fish bones. Coins are no longer accepted universally as a means of exchange in Nigeria during the period of this study, in contrast to what obtained few decades ago.

Interestingly some foreign bodies were impacted in the esophagus. While some patients volunteered the history of ingestion, others were diagnosed with plain radiographic investigations. Plain radiographs of the soft tissues of the neck, the anterior-posterior, and more importantly, the lateral view revealed radio-opaque foreign bodies within the soft tissue shadow space of the esophagus in the films. For the radio-luscent foreign bodies, the diagnosis was made with consistent air-entrapment at the same level of the esophageal soft tissue space on the radiographs. All the patients had successful removal of the FBs from the esophagus. In contrast however, patients that had FBs lodged in the larynx were diagnosed clinically from the history and clinical features at presentation. Most of these FBs were rubber/plastic toys that got impacted while the affected children were playing with their mates. Due to presentation as emergencies with rapidly developing and progressive respiratory distress, the patients had emergency tracheostomies, which proceeded to formal direct laryngoscopy, and FB removal under general anesthesia.

The only patient that had FB lodged in the bronchus was initially diagnosed to have FB in the larynx and had the above management protocol. However, at surgery no FB was found in the larynx, and further endoscopy (tracheobronchoscopy), discovered it in the right mainstem bronchus from where it was extracted. Although, $\mathrm{FB}$ in the bronchus and trachea are more common than FB in the larynx, all the cases of FB impaction in the trachea-broncheal tree that were diagnosed ab-initio were usually referred to other centers due to non -availability of bronchoscope in our center until very 
recently. This was responsible for the low proportion of trachea-broncheal FBs reported in the present study.

The complications reported in this study are in agreement with other studies ${ }^{6,12}$. Some studies from Nigeria had reported that ear nose and throat injuries were majorly caused by foreign body insertion/ ingestion and aspiration ${ }^{11,14}$. There is higher prevalence of these complications in patients whose FBs were removed by personnels who did not have adequate training in Otolaryngological practice ${ }^{3,6,12}$. Most of the cases with epistaxis were mild and resolved spontaneously. Those with secondary bacterial infection of the ear were treated with topical and systemic antibiotics while patients with fetid mucopurulent nasal discharge were treated with systemic antibiotics and nasal decongestants.

\section{Limitations}

There are some limitations in this study which should be addressed. A hospital-based study may not completely capture what happens in the community. However, almost all other studies of this nature had remained hospital based, for logistics reasons. The retrospective nature with its inherent problems including incomplete and loss of information is noted. Furthermore, the lack of comparative analysis in the distribution of the FBs between different age groupings - children vs adults vs elderly is admitted a limitation. It may be necessary to extend this study to the community level, particularly probing on the craniofacial orifices where FBs that can remain impacted for a long period of time. There is also a need to standardize treatment protocols for management of ENT FBs, in order to be able to effectively compare outcome for different studies.

\section{Conclusion}

Ear, nose and throat foreign bodies were common in North-Western Nigeria with highest incidence in children and majority were inserted into the ear. Removal attempts by untrained health professionals and the inexperienced medical personnel led to complications. The need to educate parents/guardians on close monitoring of their children to avoid such accidents was emphasized.

\section{Conflict of interests}

The authors declare that they have no conflict of interests.

\section{References}

1. Akenroye MI, OsukoyaAT. Uncommon, undeclared oesophageal foreign bodies. Niger J ClinPract 2012; 15 : 244-246.

2. Yaroko AA, Irfan M. An annual audit of the ear foreign bodies in Hospital UniversitiSains Malaysia. Malaysian Family Physician. 2012;7(1):2-5

3. Afolabi OA,Suleiman AO, Aremu SK, Eleta AP et al. An audit of paediatric nasal foreign bodies in Ilorin, Nigeria. SAJCH 2009; 3(2): 64 -67.

4. Iseh KR, Yahaya M. Ear foreign bodies: Observations on the clinical profile in Sokoto, Nigeria. Ann Afr Med 2008;7:18-23

5. Rolad NJ, McRae DRD, McCombe AW. Foreign bodies - In Key Topics in Otolaryngology and Head and Neck Surgery 2nd Edition. Bios Scientific Publisher UK 2005; 104-108

6. Fasunla J, Ibekwe T, Adeosun A: Preventable Risks in the Management of Aural Foreign Bodies in Western Nigeria. The Internet Journal of Otorhinolaryngology. 2007 7(1). DOI: 10.5580/18fe

7. Ette VF. Pattern of Ear, Nose and Throat Foreign Bodies seen in Uyo Nigeria. Ibom Medical Journal. 2012; 5(1). DOI: 41.203.67.54

8. Ogunleye AOA, Sogebi ROA.Otic foreign bodies in children in Ibadan, Nigeria. Nigerian Journal of Surgical Research 2005; 7(3): 305-308

9. Olajide TG, Ologe FE, Arigbede OO. Management of foreign bodies in the ear: a retrospective review of 123 cases in Nigeria. Ear Nose Throat J. 2011; 90(11): 16 $-19$

10. Heim SW, Maughan KL. Foreign Bodies in the Ear, Nose, and Throat. Am Fam Physician. 2007; 76(8):1185 $-1189$

11. Sogebi OA, Olaosun AO, Tobih JE, Adedeji TO, Adebola SO. Pattern of Ear, Nose and Throat Injuries in Children at LadokeAkintola University of Technology Teaching Hospital, Osogbo, Nigeria. African Journal of Paediatric Surgery 2006; 3(2) 61- 63.

12. Figueiredo RR, de Azevedo AA, de Ávila AO, Kós, Tomita S. Complications of ENT foreign bodies: a retrospective study Rev. Bras. Otorrinolaringol. 2008; 74 (1). DOI:10.1590/S0034

13. Ahmad BM, Abubakar OY. Pharyngo-oesophageal Foreign Bodies in Maiduguri. The Nigerian Journal of Surgical Research 2001; 3(2): 62 - 65

14. Aremu SK, Alabi BS, SegunBusari S, Omotoso W. Audit of Pediatric ENT Injuries. International Journal of Biomedical Science 2011; 7(3): 218-221 